\title{
LINEAR RESOLVENT GROWTH OF RANK ONE PERTURBATION OF A UNITARY OPERATOR DOES NOT IMPLY ITS SIMILARITY TO A NORMAL OPERATOR.
}

\author{
NIKOLAI NIKOLSKI AND SERGEI TREIL \\ To the memory of Tom Wolff
}

\begin{abstract}
The main result of this paper is that for any unitary (selfadjoint) operator $U$ with non-trivial absolutely continuous part of the spectrum there exists a rank one perturbation $K=b a^{*}=(\cdot, a) b$, such, that the operator $T=U+K$ satisfies the Linear Resolvent Growth condition (LRG),

$$
\left\|(\lambda I-T)^{-1}\right\| \leq C / \operatorname{dist}(\lambda, \sigma(T)), \quad \lambda \in \mathbb{C} \backslash \sigma(T),
$$

its spectrum lies on the unit circle $\mathbb{T}$ (on the real line $\mathbb{R}$ ), but $T$ is not similar to a normal operator.

This contrasts sharply with the result of M. Benamara and the first author [1] that if a finite rank perturbation $T=U+K$ of a unitary operator is a contraction $(\|T\| \leq 1)$, then it is similar to a normal operator if and only if it satisfies (LRG) and its spectrum does not cover the unit $\operatorname{disc} \mathbb{D}$.
\end{abstract}

\section{NotATion}

$\mathbb{D}$ Open unit disk in the complex plane $\mathbb{C}, \mathbb{D}:=\{z \in \mathbb{C}:|z|<1\}$; Unit circle, $\mathbb{T}:=\partial \mathbb{D}=\{z \in \mathbb{C}:|z|=1\} ;$ Upper half-plane, $\mathbb{C}_{+}:=\{z \in \mathbb{C}: \operatorname{Im} z>0\}$; Resolvent of the operator $T, R_{\lambda}^{T}:=(\lambda I-T)^{-1}$;

$a^{*}$ If $a$ is a vector in a Hilbert space, then $a^{*}:=(\cdot, a)$ stands for the linear functional (operator) $f \mapsto(f, a)$;

$b a^{*} \quad$ Stands for the operator $b a^{*}:=b(\cdot, a)$, i. e. for the operator $f \mapsto(f, a) b ;$

$H^{2}, H_{-}^{2} \quad$ Hardy class of analytic and antianalytic functions in the disk or in the upper half-plane;

$P_{+}, P_{-} \quad$ Riesz projections i. e. orthogonal projections onto $H^{2}$ and $H_{-}^{2}$ respectively;

$m \quad$ Normalized $(m(\mathbb{T})=1)$ Lebesgue measure on $\mathbb{T}$;

Date: July, 2001.

S. Treil is partially supported by the NSF grant DMS 9970395. 


\section{INTRODUCTION}

The similarity problem consists in finding criteria for a given operator $T$ in a Hilbert space $H$ to be similar to a normal operator. Recall, that an operator $N$ in a Hilbert space $H$ is called normal if $N^{*} N=N N^{*}$ and the operators $N$ and $T$ are similar if there exists a linear isomorphism $V$ such, that $T=V N V^{-1}$. As it is well known, normal operators are characterized by the existence of an orthogonal spectral resolution, or by the spectral theorem - an infinitedimensional analogue of diagonalization of hermitian matrices (see below for the von Neuman version of the spectral theorem). This characterization is the main reason why the similarity is so important: being similar to a normal operator, the given operator allows an unconditionally convergent diagonalization (instead of the orthogonal one), which is enough for most consumers of spectral theory.

The most practically interesting case arises in the perturbation theory, meaning that the operator $T$ is a "small" perturbation of a given normal operator $N_{0}, T=N_{0}+K$. Classical cases are concerned with selfadjoint or unitary $N_{0}$. It is also worth mentioning, that that, in a sense, the classical scattering theory concerns in part with similarity problem. One of the main results, the famous Kato-Rosenblum theorem states, that for a selfadjoint operator $N_{0}$ and its perturbation $N_{0}+K$ by a selfadjoint trace class operator $K$, the absolutely continuos parts of both operators are similar (and so unitarily equivalent) to each other.

For details on the long history of the problem we refer, for example, to [1], or to [11]. It is also worth mentioning that various approaches used in the field can be roughly classified as those based on Friedrichs' $\Gamma$-equations, or on J. Wermer's calculus, or on the Sz.-Nagy-Foias model representation.

In this paper, the reader can recognize some elements of the calculus approach. Namely, the basic Wermer's theorem [12] says that an operator $T$ is similar to a normal one if and only if there exists a bounded homomorphism $\mathbf{j}: C(\sigma) \rightarrow L(H)$ from the algebra $C(\sigma)$ of all continuos functions on the spectrum $\sigma=\sigma(T)$ to the algebra $L(H)$ of all bounded linear operators on $H$, such that $\mathbf{j}(z)=T$; here $z$ is the identity mapping of the complex plane, $z(\xi) \equiv \xi$ (independent variable). An immediate consequence is that an operator $T$ similar to a normal one satisfies the following Linear Resolvent Growth condition

$$
\left\|R_{\lambda}^{T}\right\|=\left\|(\lambda I-T)^{-1}\right\| \leq \frac{C}{\operatorname{dist}(\lambda, \sigma(T))}, \quad \lambda \in \mathbb{C} \backslash \sigma(T) .
$$

Of course, this observation does not depend on any theory and is a straightforward corollary of the similarity by itself: if $T=V N V^{-1}$, then

$$
\left\|R_{\lambda}^{T}\right\|=\left\|V R_{\lambda}^{N} V^{-1}\right\| \leq\|V\| \cdot\left\|V^{-1}\right\| \cdot\left\|R_{\lambda}^{N}\right\|=\|V\| \cdot\left\|V^{-1}\right\| \cdot \frac{1}{\operatorname{dist}(\lambda, \sigma(N))}
$$

and $\sigma(T)=\sigma(N)$. 
The simplest, and often the most practical test for the similarity would be the converse of the last observation. Thus, the Resolvent test Problem, as a part of Similarity Problem, is to know for what classes of $T$ or of perturbations $T=N+K$ of a "good" normal operator $N$, the above LRG condition implies that $T$ is similar to a normal operator. Such kind of results are important for applications, since LRG condition is usually easy to check.

Of course, the Linear Resolvent Growth is not sufficient for the similarity to a normal operator. The simplest example here is probably the forward shift $S$, i. e. the multiplication by the independent variable $z$ in the Hardy space $H^{2}=H^{2}(\mathbb{D}), S f(z)=z f(z)$ (equivalent definition: $S e_{n}=e_{n+1}$, $n \geq 0$, where $\left\{e_{n}\right\}_{n=0}^{\infty}$ is the standard orthonormal basis in $\ell^{2}$ ). The shift operator $S$ satisfies the LRG condition with constant 1, but it is clearly not similar to a normal operator.

This example shows, that it is reasonable to apply the Resolvent Test only to operators with "thin" spectrum, where we can hope to "probe" spectral properties of the operator using only simplest rational functions as test functions (instead of all continuous functions, as in J. Wermer's theorem).

In [1], [6], [5] several results in this direction were obtained. For example, it was proved in [1] that if a perturbation $T=U+K$ where $U$ is unitary and $\operatorname{rank} K<\infty$ is contractive (i.e. $\|T\| \leq 1$ ), then $T$ is similar to a normal operator if and only if the Linear Resolvent Growth condition (LRG) holds and the spectrum $\sigma(T)$ does not cover the unit disc $\mathbb{D}$. $^{1}$

In this paper we consider the same situation, but without the assumption $\|T\| \leq 1$. We show that in this case the situation is completely different. Namely, the following theorem holds.

Theorem 0.1. Given a unitary operator $U$ with non-trivial absolutely continuous part of the spectrum, there exists a rank one perturbation

$$
T=U-(\cdot, a) b=U-b a^{*}
$$

such, that the spectrum $\sigma(T)$ belongs to the unit circle $\mathbb{T}$, the operator $T$ satisfies the Linear resolvent Growth (LRG) condition, but $T$ is not similar to a normal (unitary) ${ }^{2}$ operator.

Remark 0.2. The above theorem also true for rank one perturbation of selfadjoint operators (with non-trivial absolutely continous part of spectrum). One can just follow the proof for unitary operators, or use the Cayley transform. We leave details to the reader.

\footnotetext{
${ }^{1} \mathrm{~A}$ simple example shows that it is possible to have $\sigma(T)=\operatorname{clos} \mathbb{D}$ for a finite rak (rank 1) perturbation of a unitary operator. Let $U$ be the bilateral shift i. e. the multiplication by the independent variable $z$ in $L^{2}(\mathbb{T})=L^{2}(\mathbb{T}, d m)$, and let $e_{n}(z)=z^{n}, n \in \mathbb{Z}$ be the standard orthonormal basis in $L^{2}(T)$. Then the operator $T=U-e_{0} e_{-1}^{*}=U-\left(, \cdot, e_{-1}\right) e_{0}$ is unitarily equivalent to the orthogonal sum of the forward shift $S$ and its adjoint $S^{*}$. So, $\sigma(T)=\operatorname{clos} \mathbb{D}$ and $T$ is clearly not similar to a normal operator.

${ }^{2}$ Any normal operator with spectrum on the unit circle is unitary.
} 
The above theorem is the main result of the paper. The cases of unitary operators $U$ with singular spectrum (continuous or discrete) represents an open problem, as well as the general question to describe all rank one perturbations of a unitary operator which are similar to a normal one. While there are several criteria of similarity to a unitary operator, see, for example, [8], in our situation (if one considers it in the spectral representation of the unitary operator $U$ ) they give rise to two weight estimates for the Hilbert Transform, and that is still an open problem. The general case, when eigenvalues appear in the complement of the unit circle is completely understood only when the resulting operator is a contraction: for general operators we do not know any results in this direction.

To complete the introduction let us recall that operators $T: H \rightarrow H$ having unitary spectrum $(\sigma(T) \subset \mathbb{T})$ and satisfying (LRG) but not similar to a unitary operator are known for a long time (see, for example, [7]), but in all these examples the "non-unitarity defects" $I-T^{*} T, I-T T^{*}$ are not controllable.

The paper is organized as follows: in Section 1 the problem is reduced to two weight estimates for the Hilbert Transform. Section 2 discusses F. Nazarov's counterexample to Sarason's conjecture, which is the basis of our counterexample. In section 3 we present the main construction, proving the theorem, stated above.

We tried to make the paper as self-contained as possible: in particular, we do not assume any familiarity with the perturbation theory. Although one can reduce the problem to the two weight estimate for the Hilbert Transform using, for example, S. Naboko's [8] criterion of similarity to a unitary operator, we do not use it: we use only elementary analytic methods.

\section{Perturbations of unitary operators}

Let $T$ be a perturbation of a unitary operator $U, T=U+K$. Then

$$
\begin{aligned}
\lambda I-T=\lambda I-U-K & =(\lambda I-U)\left(I-(\lambda I-U)^{-1} K\right) \\
& =(\lambda I-U)\left(I-R_{\lambda}^{U} K\right)
\end{aligned}
$$

We are interested in the case when $K$ is a rank one operator,

$$
K=(\cdot, a) b=b a^{*} .
$$

In this case

$$
I-R_{\lambda}^{U} K=I-(\cdot, a) R_{\lambda}^{U} b=I-\left(R_{\lambda}^{U} b\right) a^{*} .
$$

Since the operator $I-b a^{*}$ is identity on $(\mathcal{L}\{a, b\})^{\perp}$, the determinant $\operatorname{det}\left(I-b a^{*}\right)=\operatorname{det}(I-(\cdot, a) b)$ is well defined.

Lemma 1.1.

$$
\operatorname{det}\left(I-b a^{*}\right)=\operatorname{det}(I-(\cdot, a) b)=1-(b, a) .
$$


Proof. Pick an orthonormal basis in $\mathcal{L}\{a, b\}$ such that $b$ has coordinates $(\beta, 0)$ in this basis. Let $a$ has coordinates $\left(\alpha_{1}, \alpha_{2}\right)$. Then in this basis

$$
b a^{*} \mid \mathcal{L}\{a, b\}=\left(\begin{array}{c}
\beta \\
0
\end{array}\right)\left(\begin{array}{ll}
\bar{\alpha}_{1} & \bar{\alpha}_{2}
\end{array}\right)=\left(\begin{array}{cc}
\beta \bar{\alpha}_{1} & \beta \bar{\alpha}_{2} \\
0 & 0
\end{array}\right),
$$

and therefore

$$
\operatorname{det}\left(I-b a^{*}\right)=\operatorname{det}\left(\begin{array}{cc}
1-\beta \bar{\alpha}_{1} & -\beta \bar{\alpha}_{2} \\
0 & 1
\end{array}\right)=1-\beta \bar{\alpha}_{1}=1-(b, a) .
$$

Lemma 1.2. Let $K:=b a^{*}$ be a rank one operator. Operator $I-K$ is invertible if and only if $d:=\operatorname{det}(I-K)=1-(b, a) \neq 0$. In this case

$$
(I-K)^{-1}=I+\frac{1}{d} K \text {. }
$$

Proof. Note that $a^{*} b=(b, a)$, and therefore $K^{2}=b a^{*} b a^{*}=(b, a) b a^{*}=$ $(b, a) K$, and similarly $K^{n}=(b, a)^{n-1} K$. Therefore for $|\lambda|<1 /\|K\|$

$$
\begin{aligned}
(I-\lambda K)^{-1} & =I+\lambda K+\lambda^{2} K^{2}+\ldots \\
& =I+\lambda K \cdot\left(1+\lambda(b, a)+\lambda^{2}(b, a)^{2}+\ldots\right)=I+\frac{\lambda}{1-\lambda(b, a)} K .
\end{aligned}
$$

Taking analytic continuation to the point $\lambda=1$ we get

$$
(I-K)^{-1}=I+\frac{1}{1-(b, a)} K=I+\frac{1}{d} K .
$$

Proposition 1.3. Let $T=U+K$, where $K=b a^{*}$. For $\lambda \notin \sigma(U)$ let $b_{\lambda}:=R_{\lambda}^{U} b:=(\lambda I-U)^{-1} b$, and $a_{\lambda}:=\left(R_{\lambda}^{U}\right)^{*} a$, and $d(\lambda)=\operatorname{det}\left(I-b_{\lambda} a^{*}\right)=$ $1-\left(b_{\lambda}, a\right)$.

Then, if $\lambda \notin \sigma(U)$ the operator $\lambda I-T$ is invertible if and only if $d(\lambda) \neq 0$, and, moreover

$$
R_{\lambda}^{T}=(\lambda I-T)^{-1}=R_{\lambda}^{U}+\frac{1}{d(\lambda)} R_{\lambda}^{U} K R_{\lambda}^{U}=R_{\lambda}^{U}+\frac{1}{d(\lambda)} b_{\lambda} a_{\lambda}^{*} .
$$

Proof. Formula (1.1) implies that

$$
R_{\lambda}^{T}:=(\lambda I-T)^{-1}=\left(I-R_{\lambda}^{U} K\right)^{-1} R_{\lambda}^{U}
$$

and it follows from Lemma 1.2 that

$$
\left(I-R_{\lambda}^{U} K\right)^{-1}=\left(I-\left(R_{\lambda}^{U} b\right) a^{*}\right)^{-1}=I+\frac{1}{d(\lambda)} R_{\lambda}^{U} K .
$$

To prove the main theorem (Theorem 0.1) it is sufficient to consider the case when $U$ is a unitary operator with simple spectrum, so it can be realized as the multiplication by the independent variable $z$, on $L^{2}(\mu)$, where $\mu$ is a measure on the unit circle $\mathbb{T}$ (the spectral measure of $T$ ). 
Indeed, any unitary operator (in a separable Hilbert space) can be represented as an orthogonal sum of operators with simple spectrum, and to prove the theorem it is sufficient to consider just one operator in the sum.

The assumption that the operator has non-trivial absolutely continuous part of the spectrum means that for at least one operator in the sum, the corresponding measure has non-trivial absolutely continuous part.

In fact, we can without loss of generality assume that the measure $\mu$ is absolutely continuous (consider only perturbations $b a^{*}$ with with $a=b=0$ a. e. with respect to the singular part $\mu_{s}$ of the measure $\mu$ ). But for now we just assume that the operator $U$ doesn't have isolated eigenvalues, i. e. that the measure $\mu$ doesn't have isolated atoms. In this case

$$
\sigma(U)=\sigma_{\text {ess }}(U)=\operatorname{supp} \mu,
$$

where $\operatorname{supp} \mu$ stands for the closed support of the measure $\mu$, and $\sigma_{\text {ess }}$ is the essential specrum. Therefore

$$
\sigma(U)=\sigma_{\mathrm{ess}}(U)=\sigma_{\mathrm{ess}}(T) \subset \sigma(T)
$$

Since a unitary operator satisfies the linear resolvent growth condition (with constant 1), we have

$$
\left\|R_{\lambda}^{U}\right\|=\frac{1}{\operatorname{dist}(\lambda, \sigma(U))} \leq \frac{1}{\operatorname{dist}(\lambda, \sigma(T))} .
$$

So, to estimate the resolvent $R_{\lambda}^{T}$ we need to estimate

$$
\left\|R_{\lambda}^{T}-R_{\lambda}^{U}\right\|=\left\|\frac{1}{d(\lambda)} b_{\lambda} a_{\lambda}^{*}\right\|=\frac{1}{|d(\lambda)|}\left\|a_{\lambda}\right\| \cdot\left\|b_{\lambda}\right\| .
$$

It is easy to compute that

$$
b_{\lambda}=\left(R_{\lambda}^{U} b\right)(z)=\frac{b(z)}{\lambda-z}, \quad z \in \mathbb{T}
$$

and hence

$$
\left\|b_{\lambda}\right\|^{2}=\int_{\mathbb{T}} \frac{|f(z)|^{2}}{|\lambda-z|^{2}} d \mu(z)=\int_{\mathbb{T}} \frac{|f(z)|^{2}}{|1-\lambda \bar{z}|^{2}} d \mu(z)=\left.\left.|1-| \lambda\right|^{2}\right|^{-1} \mathcal{P}_{\lambda}\left(|b|^{2} d \mu\right),
$$

where $\mathcal{P}_{\lambda}(d \nu)$ denotes the Poisson (harmonic) extension of the measure $\nu$ at the point $\lambda \in \mathbb{C} \backslash \mathbb{T}$,

$$
\mathcal{P}_{\lambda}(d \nu):=\int_{\mathbb{T}} \frac{\left.|1-| \lambda\right|^{2} \mid}{|1-\lambda \bar{z}|^{2}} d \nu(z)
$$

Similarly,

$$
a_{\lambda}:=\left(\left(R_{\lambda}^{U}\right)^{*} a\right)(z)=a(z) /(\overline{\lambda-z}), \quad z \in \mathbb{T},
$$

and so

$$
\left\|a_{\lambda}\right\|^{2}=\left.\left.|1-| \lambda\right|^{2}\right|^{-1} \mathcal{P}_{\lambda}\left(|a|^{2} d \mu\right)
$$

Notice, that the determinant $d(\lambda)$ also can be easily computed:

$$
d(\lambda)=1-\left(b_{\lambda}, a\right)=1-\int_{\mathbb{T}} \frac{b(z) \overline{a(z)}}{\lambda-z} d \mu(z) .
$$


Gathering everything together we get that the linear resolvent growth condition for $T$ is equivalent to the estimate

$$
\mathcal{P}_{\lambda}\left(|a|^{2} d \mu\right) \cdot \mathcal{P}_{\lambda}\left(|b|^{2} d \mu\right) \leq C|d(\lambda)|^{2} \frac{\left(1-|\lambda|^{2}\right)^{2}}{\operatorname{dist}(\lambda, \sigma(T))^{2}}, \quad \lambda \in \mathbb{C} \backslash \sigma(T)
$$

In the case of unitary spectrum $(\sigma(T) \subset \mathbb{T})$, the last estimate can be further simplified using the following lemma by Y. Domar [3].

Lemma 1.4. Let $\sigma$ be a closed subset of the unit circle $\mathbb{T}$, and $\varphi$ be a nonnegative subharmonic function in $\mathbb{C} \backslash \sigma$ satisfying

$$
\left|\varphi(\lambda) \leq \frac{1}{\operatorname{dist}(\lambda, \mathbb{T})}=\frac{1}{|1-| \lambda||}, \quad\right| \lambda \mid \neq 1
$$

Then

$$
\varphi(\lambda) \leq \frac{C}{\operatorname{dist}(\lambda, \sigma)}, \quad \lambda \in \mathbb{C} \backslash \sigma
$$

where $C$ is some absolute constant.

This lemma, applied to the subharmonic functions $\varphi=\varphi_{x, y}, \varphi_{x, y}(\lambda)=$ $\left|\left(R_{\lambda}^{T} x, y\right)\right|$ implies that in the case of unitary spectrum the LRG condition $\left\|R_{\lambda}^{T}\right\| \leq C / \operatorname{dist}(\lambda, \sigma(T))$ follows from (and so is equivalent to) a weaker one $\left\|R_{\lambda}^{T}\right\| \leq C^{\prime} /|1-| \lambda||$. So, in the case of unitary spectrum the condition (1.3), which is equivalent to (LRG), can be replaced by

$$
\mathcal{P}_{\lambda}\left(|a|^{2} d \mu\right) \cdot \mathcal{P}_{\lambda}\left(|b|^{2} d \mu\right) \leq C|d(\lambda)|^{2}(1+|\lambda|)^{2}, \quad \lambda \in \mathbb{C} \backslash \mathbb{T} .
$$

Moreover, since for a measure $\nu$ on the circle $\mathbb{T}$ we have $\mathcal{P}_{\lambda}(d \nu)=\mathcal{P}_{1 / \lambda}(d \nu)$, the latter condition is equivalent to

$$
\mathcal{P}_{\lambda}\left(|a|^{2} d \mu\right) \cdot \mathcal{P}_{\lambda}\left(|b|^{2} d \mu\right) \leq C|d(\lambda)|^{2}, \quad \lambda \in \mathbb{D} .
$$

To prove the theorem let us now make some simplifying assumptions.

As we already discussed above, we can assume without loss of generality that our unitary operator $U$ is a multiplication by the independent variable $z$ in $L^{2}(\mu)$ where $\mu$ is an absolutely continuous (with respect to Lebesgue measure) measure on the unit circle $\mathbb{T}$.

Moreover, we can always assume (again without losing generality) that $d \mu=\chi_{E} d m$, where $E$ is a measurable set, and $m$ is the normalized $(m(\mathbb{T})=$ 1) Lebesgue measure on $\mathbb{T}$. Indeed, if $d \mu=w d m$, we put $E:=\{x \in$ $\mathbb{T}: w(x) \neq 0\}$, and it is an easy exercise to show that the operators of multiplication by $z$ in $L^{2}(\mu)$ and $L^{2}\left(\chi_{E} d m\right)$ are unitarily equivalent.

We will be considering only perturbations with the functions $a$ and $b$ having disjoint supports, i. e. satisfying $a b \equiv 0$,

In this case $d \equiv 1, \sigma(T)=\sigma(U)=\operatorname{Clos}(E) \subset \mathbb{T}$, so, the estimate (1.4) (and so (LRG)) can be rewritten as

$$
\mathcal{P}_{\lambda}\left(|a|^{2} d m\right) \cdot \mathcal{P}_{\lambda}\left(|b|^{2} d m\right) \leq C<\infty, \quad \lambda \in \mathbb{D} .
$$

Here we assume that $a$ and $b$ are supported on $E$, so we de not have to write $\chi_{E} d m$. In what follows we will even skip $d m$ and write it as $\mathcal{P}\left(|a|^{2}\right) \mathcal{P}\left(|b|^{2}\right) \leq$ C. 
Let us now find what does it mean in this case that $T$ is similar to a normal operator. First of all, since $\sigma(T) \subset \mathbb{T}$, the normal operator has to be a unitary one.

So, let us $T=V \mathcal{U} V^{-1}$, where $\mathcal{U}$ is a unitary operator. By the Spectral Theorem (see [2]), $\mathcal{U}$ can be realized as the multiplication by the independent variable $z$ in a Von Neuman integral

$$
\mathbf{H}:=\int_{\mathbb{T}} \oplus E(z) d \nu(z)
$$

where $\nu$ is a scalar spectral measure of the operator $\mathcal{U}$. Fix such a realization.

Pick $f, g \in L^{2}\left(\chi_{E} d m\right),\|f\|=\|g\|=1$ (again we can assume that $f=$ $g=0$ outside of $E$ ). Let $\tilde{f}:=V^{-1} f, \tilde{g}:=V^{*} g, \tilde{f}, \tilde{g} \in \mathbf{H}$. Then

$$
\left((\lambda I-T)^{-1} f, g\right)=\left((\lambda I-\mathcal{U})^{-1} \tilde{f}, \tilde{g}\right)=\int_{\mathbb{T}} \frac{1}{\lambda-z} \cdot(\tilde{f}(z), \tilde{g}(z))_{E(z)} d \nu(z)
$$

If we define the (complex-valued) measure $\tilde{\nu}=\tilde{\nu}_{f, g}$ by

$$
\tilde{\nu}_{f, g}(X)=\int_{X}(\tilde{f}(z), \tilde{g}(z))_{E(z)} d \nu(z),
$$

for Borel measurable subsets $X \subset \mathbb{T}$, then

$$
\left(R_{\lambda}^{T} f, g\right)=\int_{\mathbb{T}} \frac{1}{\lambda-z} d \tilde{\nu}_{f, g}(z)=-\mathcal{C}\left[\nu_{f, g}\right](\lambda)=\mathcal{C}\left[-\nu_{f, g}\right](\lambda),
$$

where $\mathcal{C}[\mu]$ stands for the Cauchy transform of a a measure $\mu$ on $\mathbb{T}, \mathcal{C}[\mu](\lambda)=$ $\int_{\mathbb{T}}(z-\lambda)^{-1} d \mu$.

Note that

$$
\begin{aligned}
\operatorname{var}\left(\tilde{\nu}_{f, g}\right) & =\int_{\mathbb{T}}\left|(\tilde{f}(z), \tilde{g}(z))_{E(z)}\right| d \nu(z) \\
& \leq \int_{\mathbb{T}}\|\tilde{f}(z)\| \cdot\|\tilde{g}(z)\| d \nu(z) \\
& \leq\left(\int_{\mathbb{T}}\|\tilde{f}(z)\|^{2} d \nu(z)\right)^{1 / 2}\left(\int_{\mathbb{T}}\|\tilde{g}(z)\|^{2} d \nu(z)\right)^{1 / 2} \\
& =\|\tilde{f}\| \cdot\|\tilde{g}\| \leq\|V\| \cdot\left\|V^{-1}\right\|,
\end{aligned}
$$

so for any $f$ and $g$ the weak resolvent $\left(R_{\lambda}^{T} f, g\right)=\left((\lambda I-T)^{-1} f, g\right)$ is the Cauchy integral of a complex measure of bounded variation.

But such Cauchy transforms admit very simple description. Since

$$
\mathcal{C}[\mu](\lambda)=\left\{\begin{array}{cc}
\sum_{n \geq 0} \widehat{\mu}(n+1) \lambda^{n}, & |\lambda|<1 \\
-\sum_{n<0} \widehat{\mu}(n+1) \lambda^{n}, & |\lambda|>1
\end{array}\right.
$$

where $\widehat{\mu}(k)$ denotes the Fourier coefficient of the measure $\mu$, $\widehat{\mu}(k):=\int_{\mathbb{T}} \bar{z}^{k} d \mu(z)$, we have

$$
\mathcal{C}[\mu](\lambda)-\mathcal{C}[\mu](1 / \bar{\lambda})=\mathcal{P}_{\lambda}(\bar{z} d \mu(z)) .
$$


Therefore an analytic function $\varphi$ in $\mathbb{C} \backslash \mathbb{T}$ is a Cauchy transform of a (complex) measure $\mu$ (of bounded variation) if and only if its harmonization $\varphi^{H}(\lambda):=\varphi(\lambda)-\varphi(1 / \bar{\lambda})$ is the Poisson extension of the measure $\bar{z} d \mu(z)$. And that happens if and only if $\sup _{0<r<1} \int_{\mathbb{T}}|\varphi(r z)| d m(z)<\infty$.

According to a Proposition 1.3, $R_{\lambda}^{T}=R_{\lambda}^{U}+b_{\lambda} a_{\lambda}^{*}$. We already know that for a unitary operator $U$ the weak resolvent $\left(R_{\lambda}^{U} f, g\right)$ is a Cauchy transform of a measure, so we only have to check $\left(b_{\lambda} a_{\lambda}^{*} f, g\right)=\left(f, a_{\lambda}\right) \cdot\left(b_{\lambda}, g\right)$.

A simple computation gives us

$$
\left(f, a_{\lambda}\right)=\int_{E} \frac{f(z) \bar{a}(z)}{\lambda-z} d m(z)=-\mathcal{C}[f \bar{a} d m](\lambda),
$$

and similarly

$$
\left(b_{\lambda}, g\right)=-\mathcal{C}[b \bar{g} d m](\lambda)
$$

(all the functions $a, b, f, g$ are supported on $E$ ).

Recall, that for $f \in L^{2}=L^{2}(\mathbb{T}, d m)$ and $\lambda \in \mathbb{D}$

$$
\mathcal{C}[f d m](\lambda)=P_{+}[\bar{z} f](\lambda), \quad \mathcal{C}[f d m](1 / \bar{\lambda})=P_{-}[\bar{z} f](\lambda),
$$

where $P_{+}$and $P_{-}$denote the Riesz projections onto $H^{2}$ and $H_{-}^{2}$ respectively: $P_{+} f=\sum_{k \geq 0} \hat{f}(k) z^{k}, P_{-}=I-P_{+}$. Of course, we extend the functions $P_{+} f$, $P_{-} f$ harmonically to $\mathbb{D}$.

Summarizing everything we get that the weak resolvent $\left(R_{\lambda}^{T} f, g\right)$ is the Cauchy transform of a complex measure (of bounded variation) if and only if the harmonic ${ }^{3}$ in the unit disk $\mathbb{D}$ function

$$
P_{+}(\bar{z} f \bar{a}) \cdot P_{+}(\bar{z} b \bar{g})-P_{-}(\bar{z} f \bar{a}) \cdot P_{-}(\bar{z} b \bar{g})
$$

is the Poisson extension of some complex measure of bounded variation on $\mathbb{T}$.

So, to show that Linear Resolvent Growth doesn't imply similarity to a normal operator it is enough to find 4 functions $a, b, f, g \in L^{2}$, all supported by the set $E$ satisfying $a b \equiv 0$ and

$$
\mathcal{P}_{\lambda}\left(|a|^{2}\right) \cdot \mathcal{P}_{\lambda}\left(|b|^{2}\right) \leq C
$$

and such, that the harmonic function $P_{+}(a f) \cdot P_{+}(b g)-P_{-}(a f) \cdot P_{-}(b g)$ is not a harmonic extension of a (complex) measure of bounded variation. To avoid unnecessary notation we have replaced here $\overline{z g}$ by $g$ and $\overline{z a}$ by $a$.

To construct such functions we will use Nazarov's counterexample to a Sarason's conjecture about two weight estimates for Hilbert Transform.

\section{Nazarov's counterexample to Sarason's Conjecture.}

In [9] F. Nazarov proved the following result. Let $\mathcal{H}$ denote the Hilbert Transform

$$
\mathcal{H} f(y)=\frac{1}{\pi} \int_{\mathbb{R}} \frac{f(x)}{y-x} d x .
$$

\footnotetext{
${ }^{3}$ The first term in the function is a product of two analytic ones, and the second is the product of two antianalytic, so the function is indeed harmonic
} 
Let $a, b$ be functions on $\mathbb{R}$. We are interested when the operator $M_{a} \mathcal{H} M_{b}$, where $M_{a}, M_{b}$ are multiplication operators by the functions $a$ and $b$ respectively, is a bounded operator on $L^{2}$. It was conjectured by D. Sarason, that the operator $M_{a} \mathcal{H} M_{b}$ is bounded if and only if

$$
\mathcal{P}_{\lambda}\left(|a|^{2}\right) \cdot \mathcal{P}_{\lambda}\left(|b|^{2}\right) \leq C<\infty \quad \lambda \in \mathbb{C}_{+} ;
$$

here for a function $\varphi$ defined on $\mathbb{R}$ the symbol $\mathcal{P}_{\lambda}(\varphi)$ stands for its harmonic extension at the point $\lambda \in \mathbb{C}_{+}$.

The above condition (2.1) is indeed necessary for the boundedness of the operator $M_{a} \mathcal{H} M_{b}$. But, as the following result by F. Nazarov asserts, this condition is not sufficient.

Theorem 2.1. There exist functions $a, b$ satisfying (2.1) and such, that the operator $M_{a} \mathcal{H} M_{b}$ is not bounded on $L^{2}$.

Now let us make several simple observations. First of all

$$
\left\|M_{a} \mathcal{H} M_{b}\right\|=\left\|M_{|a|} \mathcal{H} M_{|b|}\right\|,
$$

i. e. the norm depends only on absolute values of $a$ and $b$.

The second observation is that one can replace the Hilbert Transform $\mathcal{H}$ by the Riesz projection $P_{+}$, where $P_{+}$is the orthogonal projection onto $H^{2}=H^{2}\left(\mathbb{C}_{+}\right)$. Indeed, $P_{+}=\frac{1}{2}(I+i \mathcal{H})$ and condition (2.1) implies that $a b \in L^{\infty}$, and therefore the operator $M_{a b}=M_{a} M_{b}$ is bounded.

Third observation is that it doesn't matter if we work on the real line or on the unit circle $\mathbb{T}$. Indeed, let us consider conformal mapping $\tau$ from $\mathbb{C}_{+}$ onto the unit disk $\mathbb{D}, \tau(z)=\frac{i-z}{i+z}$, and let $\mathcal{U}: L^{2}(\mathbb{T}) \rightarrow L^{2}(\mathbb{R})$ be a unitary mapping, $\mathcal{U} f:=\frac{1}{\sqrt{\pi}} \frac{1}{x+i} f(\tau(x))$. Clearly (and it is a very well known fact), $\mathcal{U} H^{2}(\mathbb{D})=H^{2}\left(\mathbb{C}_{+}\right)$, and

$$
\mathcal{U}^{-1} M_{a} P_{+}^{\mathbb{C}_{+}} M_{b} \mathcal{U}=M_{a_{1}} P_{+}^{\mathbb{D}} M_{b_{1}},
$$

where $a_{1}:=a \circ \tau^{-1}, b_{1}:=b \circ \tau^{-1}$. Since the condition (2.1) is conformally invariant, $a, b$ satisfy it if and only if

$$
\sup _{\lambda \in \mathbb{D}} \mathcal{P}_{\lambda}\left(\left|a_{1}\right|^{2}\right) \cdot \mathcal{P}_{\lambda}\left(\left|b_{1}\right|^{2}\right)<\infty
$$

And final observation is that given an interval (an arc) $\mathcal{I} \subset \mathbb{T}$ one can find functions $a, b$ satisfying (2.1), supported by $\mathcal{I}$ and such, that the operator $M_{a} P_{+} M_{b}$ is not bounded. This is easier to see on the circle $\mathbb{T}$. Take the functions $a, b$ on the circle $\mathbb{T}$, and consider a smooth finite partition of unity $1=\sum_{1}^{n} \varphi_{k}, 0 \leq \varphi_{k} \leq 1$, such that each $\varphi_{k}$ is supported by a closed interval of length at most $|\mathcal{I}| / 2$. Then

$$
M_{a} P_{+} M_{b}=\sum_{1 \leq j, k \leq n} M_{a \varphi_{k}} P_{+} M_{b \varphi_{j}}
$$

so at least one of the operators $M_{a \varphi_{k}} P_{+} M_{b \varphi_{j}}$ is unbounded. This can happen only if (closed) supports of $\varphi_{k}$ and $\varphi_{j}$ have nontrivial intersection. Therefore 
both functions $\varphi_{k} a$ and $\varphi_{j} b$ are supported by one interval of length at most $|\mathcal{I}|$, and rotating the picture completes the proof.

So, we get the result we will be using.

Theorem 2.2. Given an arc $\mathcal{I} \subset \mathbb{T}$ there exist functions $a, b \in L^{2}=L^{2}(\mathbb{T})$ supported by $\mathcal{I}$, and satisfying

$$
\sup _{\lambda \in \mathbb{D}} \mathcal{P}_{\lambda}\left(|a|^{2}\right) \cdot \mathcal{P}_{\lambda}\left(|b|^{2}\right)<\infty,
$$

and such, that the operator $M_{a} P_{+} M_{b}$ is not bounded on $L^{2}$.

Remark 2.3. The fact that $a, b \in L^{2}=L^{2}(\mathbb{T})$ follows immediately from (2.2).

\section{Constructing the Counterexample}

Consider a sequence of $\operatorname{arcs} \mathcal{I}_{n} \subset \mathbb{T},\left|\mathcal{I}_{n}\right|=2^{-n-5}$ such that the $\operatorname{arcs} 10 \mathcal{I}_{n}$ are disjoint. Here, given an interval (arc) $\mathcal{I}$ we denote by $k \mathcal{I}$ the interval (arc) with the same center and of length $k \cdot|\mathcal{I}|$.

Let $\mu$ be a complex measure (of bounded variation) on $\mathbb{T}$, and let $\Phi=\Phi_{\mu}$ be it harmonic extension into the unit disk $\mathbb{D}$. It is an easy exercise to check that for any sequence $r_{n}, 1-\left|\mathcal{I}_{n}\right| \leq r_{n}<1$

$$
\sum_{n=1}^{\infty} \int_{2 \mathcal{I}_{n}}\left|\Phi\left(r_{n} \theta\right)\right| d m(\theta) \leq C \operatorname{var} \mu
$$

with some constant $C$, not depending on $\mathcal{I}_{n}, r_{n}, \mu$ as long as they satisfy the above assumptions.

Now let us describe the main component of the construction. Let $E$ be a subset of $\mathbb{T}$ of positive measure. Recall, that a point of $x \in E$ is called a (Lebesgue) density point if

$$
\lim _{|\mathcal{I}| \rightarrow 0, \mathcal{I} \ni x} \frac{m(E \cap \mathcal{I})}{m(\mathcal{I})}=1,
$$

and that almost all points of $E$ are density points.

Proposition 3.1. Given a density point $x_{0} \in E$ and numbers $R, \varepsilon, \delta>0$, one can find an arbitrary small arc $\mathcal{I} \subset \mathbb{T}(|\mathcal{I}|<\delta)$ centered at $x_{0}$ and bounded functions $a, b, f, g$ supported by $\mathcal{I} \cap E$, such that $a b \equiv 0$,

$$
\sup _{\lambda \in \mathbb{C}_{+}} \mathcal{P}_{\lambda}\left(|a|^{2}\right) \mathcal{P}_{\lambda}\left(|b|^{2}\right) \leq 1, \quad\|f\|_{2} \leq \varepsilon, \quad\|g\|_{2} \leq \varepsilon,
$$

and such, that for some $r, 1-|\mathcal{I}| \leq r<1$ we have for the functions $F:=P_{+}(a f) P_{+}(b g), G:=P_{-}(a f) P_{-}(b g)$

$$
\int_{2 \mathcal{I}}|F(r \theta)| d m(\theta)>R, \quad \int_{2 \mathcal{I}}|G(r \theta)| d m(\theta)<\varepsilon .
$$


Proof. Let $\mathcal{I}$ be a small arc $(|\mathcal{I}|<\delta)$ centered at $x$. By Theorem 2.2 there exist functions $a, b \in L^{2}$ supported by the interval $\mathcal{I}$ such that the operator $M_{b} P_{+} M_{a}$ is unbounded. Then there exists a function $f \in L^{2}$ such that $b P_{+}(a f) \notin L^{2}$. That means for some $g \in L^{2}$ the function $b g P_{+}(a f)$ is not in $L^{1}$. So we can pick bounded $\left(L^{\infty}\right)$ functions $a, b, f, g$ supported by the interval $\mathcal{I}$, satisfying (3.1) and such, that $\left\|b g P_{+}(a f)\right\|_{1}>4 R$. Replacing $g$ by $z^{n} g(z)$, where $n>0$ is sufficiently large number we can make the norm $\left\|P_{-}(b g)\right\|_{2}$ as small as we want, so the norms $\left\|P_{-}(b g) P_{+}(a f)\right\|_{1}$, $\left\|P_{-}(b g) P_{-}(a f)\right\|_{1}$ can be as small as we want, and the norm $\left\|P_{+}(b g) P_{+}(a f)\right\|_{1}$ can be as close to the norm $\left\|b g P_{+}(a f)\right\|_{1}$ as we want. So we can always achieve

$$
\left\|P_{+}(a f) P_{+}(b g)\right\|_{1}>3 R, \quad\left\|P_{-}(a f) P_{-}(b g)\right\|_{1}<\varepsilon / 2 .
$$

Now we want all the functions to be supported by $\mathcal{I} \cap E$. Since all functions are bounded, so we can assume that

$$
\|a\|_{\infty},\|b\|_{\infty} \leq C, \quad\|f\|_{\infty},\|g\|_{\infty} \leq C / m(\mathcal{I})^{1 / 2}
$$

Then there exists a small constant $\alpha>0$ (depending on $C$ ) such that if $m(\mathcal{I} \backslash E)<\alpha m(\mathcal{I})$, then

$$
\left\|P_{+}\left(\chi_{E} a f\right) P_{+}\left(\chi_{E} b g\right)\right\|_{1}>2 R, \quad\left\|P_{-}\left(\chi_{E} a f\right) P_{-}\left(\chi_{E} b g\right)\right\|_{1}<\varepsilon
$$

(the functions $\chi_{E} a f, \chi_{E} b g$ are close in $L^{2}$-norm to af and $b g$ respectively). So, if $m(\mathcal{I} \backslash E)<\alpha m(\mathcal{I})$ we can just multiply all the functions by $\chi_{E}$.

It looks like a bad circle here, since we first picked the interval $\mathcal{I}$, then the functions $a, b, f, g$ (and so the constant $C$ ), and now we want $m(\mathcal{I} \backslash$ $E) / m(\mathcal{I})<\alpha=\alpha(C)$. However, we can break this bad circle, because everything can be rescaled.

This can be better explained on the real line $\mathbb{R}$. Recall, that, as we already discussed above in Section 2, we can use conformal mapping from the disk to the upper half-plane to move freely from the circle to the real line and back. So let everything is on the real line. Without loss of generality we can assume that our density point $x_{0}$ is the origin 0 .

Since the Riesz Projection $P_{+}$commutes with the scaling operators $f(z) \mapsto f(k z)$ (for all $k \in \mathbb{R} \backslash\{0\}$ ), nothing changes when we rescale the picture, i. e. replace $a(x), b(x)$ by $a(k x), b(k x)$ and $f(x), g(x)$ by $k^{1 / 2} f(k x)$, $k^{1 / 2} g(k x)$ respectively. Nothing, except the fact that the interval $\mathcal{I}$ will be replaced by $k^{-1} \mathcal{I}$, so now we can make $m(\mathcal{I} \backslash E) / m(\mathcal{I})$ as small as we want, because 0 is a density point of $E$.

Now let us pick radius $r \geq 1-|\mathcal{I}|$ sufficiently close to 1 such that for functions $F:=P_{+}(a f) P_{+}(b g b), G:=P_{-}(a g) P_{-}(f b)$ we have

$$
\int_{2 \mathcal{I}}|F(r \theta)| d m(\theta)>3 R, \quad \int_{2 \mathcal{I}}|G(r \theta)| d m(\theta)<\varepsilon / 2 .
$$

We are almost done, the only problem is that $a b \not \equiv 0$. 
To correct that, define

$$
\chi\left(e^{i t}\right)= \begin{cases}1, & 0 \leq t<\pi \\ 0 & \pi \leq t<2 \pi\end{cases}
$$

and let $\chi_{n}(z):=\chi\left(z^{n}\right)$. Our claim is, that if we replace $a$ by $\chi_{n} a$ and $b$ by $\left(1-\chi_{n}\right) b$ with sufficiently large $n$, then we are done.

Indeed, the functions $\chi_{n},\left(1-\chi_{n}\right)$ converge weakly in $L^{2}$ to the function $\frac{1}{2}$ as $n \rightarrow \infty$ (the sequence $\chi_{n}$ is bounded, and $\int f \chi_{n} d m \rightarrow \frac{1}{2} \int f d m$ for smooth $f$ ). Since for a function $\varphi \in L^{2}$,

$$
P_{+} \varphi(z)=\frac{1}{2 \pi i} \int_{\mathbb{T}} \frac{\varphi(\xi)}{\xi-z} d \xi
$$

one can conclude that the functions $F_{n}:=P_{+}\left(\chi_{n} a g\right) P_{+}\left(\left(1-\chi_{n}\right) b g\right)$ converge to $\frac{1}{2} F$ uniformly on compact subsets of $\mathbb{D}$.

Similarly, the functions $G_{n}:=P_{-}\left(\chi_{n} a f\right) P_{-}\left(\left(1-\chi_{n}\right) b g\right)$ converge to $\frac{1}{2} G(z)$ uniformly on compact subsets of $\mathbb{D}$. So, for sufficiently large $n$,

$$
\int_{2 \mathcal{I}}\left|F_{n}(r \theta)\right| d m(\theta)>R, \quad \int_{2 \mathcal{I}}\left|G_{n}(r \theta)\right| d m(\theta)<\varepsilon .
$$

Replace $a$ by $\chi_{n} a$ and $b$ by $\left(1-\chi_{n}\right) b$ with sufficiently large $n$, then we are done!

Using Proposition 3.1 take the sequence of intervals $\mathcal{I}_{n} \subset \mathbb{T}$ as above, and construct bounded functions $a_{n}, b_{n}, f_{n}, g_{n}$ supported by $\mathcal{I}_{n} \cap E$ and radii $r_{n}, 1-\left|\mathcal{I}_{n}\right| \leq r_{n}<1$ such, that

1. $a_{n} b_{n} \equiv 0$

2. $\mathcal{P}_{\lambda}\left(\left|a_{n}\right|^{2}\right) \mathcal{P}_{\lambda}\left(\left|b_{n}\right|^{2}\right) \leq 1$ for all $\lambda \in \mathbb{D}$;

3. $\left\|f_{n}\right\|_{2} \leq 2^{-n},\left\|g_{n}\right\|_{2} \leq 2^{-n}$;

and such that for the functions $F_{n}:=\left(P_{+}\left(a_{n} f_{n}\right) P_{+}\left(b_{n} g_{n}\right)\right.$, $G_{n}:=\left(P_{-}\left(a_{n} f_{n}\right) P_{-}\left(b_{n} g_{n}\right)\right.$ we have

$$
\text { 4. } \int_{2 \mathcal{I}_{n}}\left|F_{n}\left(r_{n} \theta\right)\right| d m(\theta)>2^{n}, \quad \int_{2 \mathcal{I}_{n}}\left|G_{n}\left(r_{n} \theta\right)\right| d m(\theta)<2^{-n} .
$$

The condition 2 implies that

$$
\frac{1}{\left|\mathcal{I}_{n}\right|} \int_{\mathcal{I}_{n}}\left|a_{n}\right|^{2} d m \cdot \frac{1}{\left|\mathcal{I}_{n}\right|} \int_{\mathcal{I}_{n}}\left|b_{n}\right|^{2} d m \leq C
$$

where $C<\infty$ is some absolute constant, so we can always assume that

5. $\frac{1}{\left|\mathcal{I}_{n}\right|} \int_{\mathcal{I}_{n}}\left|a_{n}\right|^{2} d m \leq C, \quad \frac{1}{\left|\mathcal{I}_{n}\right|} \int_{\mathcal{I}_{n}}\left|b_{n}\right|^{2} d m \leq C$.

Define

$$
a=\sum a_{n} \quad b:=\sum b_{n}, \quad f:=\sum f_{n}, \quad g=\sum g_{n} .
$$

It is easy to check that the pair $a, b$ satisfies the condition (2.1). Indeed, if $\operatorname{dist}\left(\lambda, \mathcal{I}_{k}\right) \geq\left|\mathcal{I}_{k}\right|$ for all $k$, then, computing $\mathcal{P}_{\lambda}\left(|a|^{2}\right)$, we can replace each 
$\left|a_{k}\right|^{2}$ on the interval $\mathcal{I}_{k}$ by its average, and since all the averages are bounded (condition 5), we get $\mathcal{P}_{\lambda}\left(|a|^{2}\right) \leq C<\infty$, and the same for $\mathcal{P}_{\lambda}\left(|b|^{2}\right)$.

If $\operatorname{dist}\left(\lambda, \mathcal{I}_{k}\right)<\left|\mathcal{I}_{k}\right|$ for some $k$, then $\operatorname{dist}\left(\lambda, \mathcal{I}_{j}\right) \geq\left|\mathcal{I}_{k}\right|$ for $j \neq k$. We can estimate

$$
\mathcal{P}_{\lambda}\left(|a|^{2}\right)=\mathcal{P}_{\lambda}\left(\left|a_{k}\right|^{2}\right)+\mathcal{P}_{\lambda}\left(\sum_{j \neq k}\left|a_{j}\right|^{2}\right) \leq \mathcal{P}_{\lambda}\left(\left|a_{k}\right|^{2}\right)+C \cdot(1-|\lambda|) /\left|\mathcal{I}_{k}\right|,
$$

and similarly for $\mathcal{P}_{\lambda}\left(\left|b_{k}\right|^{2}\right)$,

$$
\mathcal{P}_{\lambda}\left(|b|^{2}\right) \leq \mathcal{P}_{\lambda}\left(\left|b_{k}\right|^{2}\right)+C \cdot(1-|\lambda|) /\left|\mathcal{I}_{k}\right| .
$$

Recall, that the Poisson kernel $P_{\lambda}$ is given by

$$
P_{\lambda}(z)=\frac{1-|\lambda|^{2}}{|1-\bar{\lambda} z|^{2}},
$$

so $(1-|\lambda|) \cdot P_{\lambda}(z) \leq 1$. Therefore

$$
\mathcal{P}_{\lambda}\left(\left|a_{k}\right|^{2}\right) \cdot(1-|\lambda|) /\left|\mathcal{I}_{k}\right| \leq C \cdot\left|\mathcal{I}_{k}\right|^{-1} \int_{\mathcal{I}_{k}}\left|a_{k}\right|^{2} d m \leq C .
$$

and similarly $\mathcal{P}_{\lambda}\left(\left|a_{k}\right|^{2}\right) \cdot(1-|\lambda|) /\left|\mathcal{I}_{k}\right| \leq C$. The other two terms in the product

$$
\left(\mathcal{P}_{\lambda}\left(\left|a_{k}\right|^{2}\right)+C \cdot(1-|\lambda|) /\left|\mathcal{I}_{k}\right|\right)\left(\mathcal{P}_{\lambda}\left(\left|b_{k}\right|^{2}\right)+C \cdot(1-|\lambda|) /\left|\mathcal{I}_{k}\right|\right)
$$

are also trivially bounded, so indeed $\mathcal{P}_{\lambda}\left(|a|^{2}\right) \mathcal{P}_{\lambda}\left(|b|^{2}\right) \leq C<\infty$.

Let us check that the harmonic function

$$
F-G=P_{+}(a f) P_{+}(b g)-P_{-}(a f) P_{-}(b g)
$$

is not a harmonic extension of a complex measure $\mu$ of bounded variation. Formula (3.2) implies that for $z \in \mathbb{D}$, $\operatorname{dist}\left(z, \mathcal{I}_{k}\right) \geq\left|\mathcal{I}_{k}\right|$ we have the estimate

$$
\begin{aligned}
\left|P_{+}\left(a_{k} f_{k}\right)(z)\right| \leq \frac{C}{\left|\mathcal{I}_{k}\right|}\left\|a_{k} f_{k}\right\|_{1} & \leq \frac{C}{\left|\mathcal{I}_{k}\right|}\left\|a_{k}\right\|_{2} \cdot\left\|f_{k}\right\|_{2} \\
& \leq \frac{C}{\left|\mathcal{I}_{k}\right|}\left|\mathcal{I}_{k}\right|^{1 / 2} 2^{-k} \leq C 2^{-k / 2}
\end{aligned}
$$

and similar estimate holds for $\left|P_{+}\left(b_{k} g_{k}\right)\right|$. Therefore, for $\theta \in \mathcal{I}_{n}$,

$$
\sum_{k \neq n}\left|\left(P_{+}(a f) P_{+}(b g)\right)\left(r_{n} \theta\right)\right| \leq C \sum_{k=1}^{\infty} 2^{-k} \leq C,
$$

and similarly

$$
\sum_{k \neq n}\left|\left(P_{-}(a f) P_{-}(b g)\right)\left(r_{n} \theta\right)\right| \leq C .
$$

Therefore for the functions $F_{k}:=\left(P_{+}\left(a_{k} f_{k}\right) P_{+}\left(b_{k} g_{k}\right)\right.$, $G_{k}:=\left(P_{-}\left(a_{k} f_{k}\right) P_{-}\left(b_{k} g_{k}\right)\right.$

$$
\sum_{k \neq n} \int_{2 \mathcal{I}_{n}}\left|F_{k}\left(r_{n} \theta\right)\right| d m(\theta) \geq 2^{n}-C 2^{-n}
$$


and

$$
\sum_{k \neq n} \int_{2 \mathcal{I}_{n}}\left|G_{k}\left(r_{n} \theta\right)\right| d m(\theta) \leq 2^{-n}+C 2^{-n}
$$

Therefore for $F=P_{+}(a f) P_{+}(b g)=\sum_{n} F_{n}$ and $G_{n}=P_{-}(a f) P_{-}(b g)=$ $\sum_{n} G_{n}$, we have

$$
\sum_{n} \int_{2 \mathcal{I}_{n}}\left|F\left(r_{n} \theta\right)-G\left(r_{n} \theta\right)\right| d m(\theta)=+\infty
$$

Therefore, $F-G$ cannot be the harmonic extension of a (complex) measure of bounded variation, so we got a counterexample.

\section{REFERENCES}

[1] N. E. Benamara and N. K. Nikolski, Resolvent tests for similarity to a normal operator, Proc. London. Math. Soc., 78(1999), no.3, pp. 585-626.

[2] M.S. Birman and M.Z. Solomjak, Spectral theory of self-adjoint operators in Hilbert space, D. Reidel, Dordrecht-Boston-Lancaster-Tokyo, 1987.

[3] Y. Domar, On the existence of a large subharmonic minorant of a given function, Ark. Mat., 3 (1957), 429-440.

[4] N. Dunford and J. T. Schwartz, Linear operators. Part I: General theory., John Wiley \& Sons, New York etc, 1988, 858 pp.

[5] S. Kupin, Linear resolvent growth test for similarity of a weak contraction to a normal operator, Ark. Mat. 39 (2001), no. 1, 95-119.

[6] S. Kupin and S. Treil Linear resolvent growth of a weak contraction does not imply its similarity to a normal operator, Illinois Journal of Mathematics, 45 (2001), no. 1, 229-242.

[7] A. S. Markus, Some tests for the completeness of the system of root vectors of a linear operator in a Banach space, Mat. Sb (N.S.), 70 (1966), 526-565 (Russian); English translation: Amer. Math. Soc. Transl., 85 (1969), No 2.

[8] S. Naboko, Conditions for similarity to unnitary and selfadjoint operators, Funkcional. Anal. i Prilozhen., 18 (1984), 16-27 (Russian); English translation in: Funct. Anal. Appl., 18 (1984), 13-22.

[9] F. Nazarov, A counterexample to Sarason's conjecture, Preprint, MSU, 1997, 19 pp; Available at http://www.math.msu.edu/ fedja/prepr.html

[10] N. Nikolski and S. V. Khruschev, A function model and some problems in spectral function theory, Trudy Math. Inst. Steklov 176(1987), pp. 97-210 (Russian); English transl. in: Proc. Steklov Inst. Math. 176(1988), no.3, pp. 111-214.

[11] N. Nikolski and V. Vasyunin, Elements of spectral theory in terms of the free function model. Part I: Basic constructions, in: Holomorphic spaces (eds. Sh. Axler, J. McCarthy and D. Sarason), MSRI publications, 33 (MSRI, Berkeley, 1998), pp. 211-302.

[12] J. Wermer, Commuting spectral operators on Hilbert space, Pacific J. Math., 4 (1954), 335-361.

Nikolski: Laboratoire de Mathématiques Pures, Université Bordeaux 1, 351 Cours de la Libération, 33405 Talence, France

E-mail address: nikolski@math.u-bordeaux.fr

Treil: Department of Mathematics, Brown Univrsity, 151 Thayer Str./Box 1917, Providence, RI 02912, USA

E-mail address: treil@math.brown.edu

$U R L:$ http: //www . math. brown. edu/ ${ }^{\sim}$ treil 